\title{
Una experiencia de formación para futuros profesores: producir matemática en un contexto de modelización matemática vinculada con fenómenos geométricos
}

\section{A Teaching Experience for Prospective Teachers: to Produce Mathematics in a Context of Mathematical Modelling Connected with Geometrical Phenomenon}

\author{
María Florencia Cruz ${ }^{1}$ \\ Cristina Esteley ${ }^{2}$ \\ Sara Scaglia ${ }^{3}$
}

\begin{abstract}
Resumen: En este artículo presentamos una propuesta de enseñanza que se focaliza en objetos geométricos y se pone en juego con 17 estudiantes que cursan en 2018 una asignatura de geometría del tercer año del programa de formación de profesores en matemática de la Universidad Nacional del Litoral de Santa Fe-Argentina.

Diseñamos y organizamos la propuesta en torno a procesos de modelización matemática, buscando que los estudiantes reproduzcan en el aula actividades propias del quehacer matemático. Las consignas diseñadas para el trabajo de los estudiantes toman como recurso imágenes de figuras tridimensionales.

Cabe indicar que la propuesta y su puesta en aula forman parte de un trabajo de tesis de doctorado en educación matemática en la que se estudia una experiencia educativa con futuros profesores cuando trabajan en un contexto de modelización matemática. Para el presente artículo describimos y analizamos en
\end{abstract}

Fecha de recepción: 28 de mayo de 2019. Fecha de aceptación: 16 de enero de 2020.

1 Facultad de Humanidades y Ciencias. Universidad Nacional del Litoral. ma.florenciacruz@gmail.com, orcid.org/0000-0002-9184-6292.

2 Facultad de Matemática, Astronomía, Física y Computación. Universidad Nacional de Córdoba. esteley@famaf.unc.edu.ar, orcid.org/0000-0001-5114-8054.

3 Facultad de Humanidades y Ciencias. Universidad Nacional del Litoral. sbscaglia@gmail.com, orcid. org/0000-0002-4935-2379. 
detalle la propuesta y las principales ideas que la sustentan. También discutimos algunos avances vinculados con las producciones emergentes del trabajo de los estudiantes. Finalmente ofrecemos algunas conclusiones.

Palabras Clave: Formación de Profesores, Modelización Matemática, Geometría, Validación Matemática, Modelo.

\begin{abstract}
In this article we present a teaching proposal that focuses on geometric objects that is carried out in 2018 with 17 prospective teachers. At that time, they are taking a geometry course corresponding to the third year of the formation program for teachers in mathematics of the Universidad Nacional del Litoral de Santa Fe - Argentina.

We design and organize the main moments of the proposal around mathematical modelling processes so that prospective teachers are engaged in characteristic activities of mathematical production. The major task designed for the students' work takes as its main resource printed images of three-dimensional figures.

It is worth noting that the proposal is designed and implemented as part of research associated with a doctoral thesis in mathematics education. In order to emphasize the pedagogical aspect of the implementation of the research work related to the thesis, in this article we describe and analyse in detail the educational proposal. We advance in discussions about the students' productions that emerge from their interactions in classes. Finally, we and offer some conclusions.
\end{abstract}

Keywords: Teachers Professional Development, Mathematical Modelling, Geometry, Mathematical Validation, Model.

\title{
1. INTRODUCCIÓN
}

Este artículo tiene como propósito dar a conocer y discutir una experiencia de formación para futuros profesores en matemática en un contexto de modelización matemática (MM). Su diseño y ejecución toma como sustento avances de una investigación que persigue por objetivo general estudiar conocimientos puestos en juego por futuros profesores en matemática de la Facultad de Humanidades y Ciencias de la Universidad Nacional del Litoral (UNL), al trabajar en 
un contexto educativo mediado por actividades de modelización matemática en un curso de geometría. Particularmente, en la investigación interesa indagar los procesos de validación y los sentidos atribuidos a esos procesos por los futuros profesores en el marco de la experiencia de modelización matemática.

El objetivo de la investigación entrelaza temáticas fundamentales en el ámbito de la educación matemática: la formación de futuros profesores, el trabajo con MM y la validación. Estas temáticas se tratan en diversos grupos de discusión en ocasión del 13 Congreso Internacional de Educación Matemática (ICME) celebrado en 2016 y en la 17 Conferencia Internacional sobre la Enseñanza de la Modelización Matemática y sus Aplicaciones (ICTMA) celebrada en 2015. Algunas producciones que dan cuenta del avance y preocupación de estas temáticas son Stylianides y Harel (2018), Stillman, Blum y Kaiser (2017), Kaiser (2017), Hanna y De Villiers (2012), y Reid y Knipping(2010).

Para responder al objetivo general vinculado con la investigación antes mencionada apelamos a un abordaje de naturaleza cualitativa, particularmente a una investigación de diseño (Bakker y van Eerde, 2015) en la cual se emplea un conjunto de tareas secuenciadas y novedosas, se busca conocer recursos y conocimientos previos puestos en juego por estudiantes, modos en que se crean registros, formas en que surgen concepciones y sus cambios, recursos que se emplean, entre otros (Confrey, 2006).

Atendiendo a las consideraciones metodológicas realizadas diseñamos una propuesta que tiene dos finalidades, una de índole investigativa y otra pedagógica relativa al quehacer del trabajo matemático en un curso de Geometría Euclídea Espacial (GEE). Para el diseño de la propuesta de enseñanza consideramos, entre otros, literatura vinculada con la temática en estudio y resultados de entrevistas realizadas con futuros profesores que cursaron GEE en 2017. Estas últimas nos permiten conocer recursos y conocimientos previos de los estudiantes ${ }^{4}$ y concluimos que posiblemente no han tenido oportunidad en su formación de vivir experiencias educativas en las que se apela a MM (Cruz, Esteley y Scaglia, 2018).

Tanto la investigación como este artículo que focaliza en la propuesta, se conectan con cuestiones relativas a formación de futuros profesores en matemática. Esta área posee actualmente interés en el ámbito internacional (Even y Ball, 2009), señalamos los resultados del Survey presentado en el último Congreso Internacional de Educación Matemática (ICME) (Robutti, et al., 2016) y la convocatoria para la próxima Conferencia de Estudio número 25 (ICMI Study

\footnotetext{
${ }^{4}$ En este texto al mencionar el término estudiantes se refiere a futuros profesores en matemática.
} 
25) a realizarse en febrero de 2020 (Borko, et al., 2019). A su vez, la preocupación respecto a la formación matemática de jóvenes de los distintos niveles educativos pone de manifiesto la necesidad de contribuir y prestar especial atención a la formación docente de quienes enseñarán matemática. En Argentina, la propuesta de estándares creada por el Consejo Interuniversitario Nacional para las carreras de profesorado universitario ${ }^{5}$ en matemática (2012), explicita la necesidad de que futuros profesores trabajen con "procedimientos tales como: [...] ejemplificación, validación, contrastación [...], elaboración de conjeturas, modelización y visualización" (pp. 6 y 7).

Recuperando las recomendaciones internacionales y locales, la propuesta de enseñanza diseñada apela a MM como abordaje pedagógico en el sentido de Bassanezi (1994) y Esteley (2014). Esto supone la organización del trabajo en el aula de matemática en torno a las fases que intervienen en un proceso de modelización de naturaleza matemática. Al respecto, Esteley (2014) afirma que "la modelización matemática como abordaje pedagógico se refiere básicamente al trabajo con modelos matemáticos en el aula e intenta reproducir en las mismas, las actividades de la comunidad matemática" (p.1). Cabe indicar que MM como abordaje pedagógico guarda algunas similitudes con resolución de problemas, pero también presentan diferencias ${ }^{6}$ (Blum y Niss, 1991; Esteley, 2014).

Para la propuesta de enseñanza se recuperan las ideas de MM y validación en formación de profesores. En la puesta en aula, en el marco de la asignatura GEE, emerge principalmente el trabajo matemático sobre el estudio de figuras tridimensionales y en particular la noción de poliedro. Este contenido resulta de interés en la formación de profesores, dado que forma parte de la propuesta curricular para la escolaridad obligatoria en el sistema educativo argentino (NAP, 2011; Diseño Curricular Educación Secundaria Orientada de la Provincia de Santa Fe, 2014).

Con una propuesta pensada desde la MM enlazada con fenómenos geométricos, se busca ir más allá de la enseñanza tradicional de la matemática posibilitando a los estudiantes el contacto con una experiencia de construcción de conocimiento y de reflexión acerca de lo construido. En sinergia con lo propuesto por Blum (2015),

\footnotetext{
5 Universidades argentinas ofrecen carreras de grado para la formación de Profesores en Matemática. Los profesores que egresan de esos programas están habilitados para desempeñar en colegios secundarios y en la universidad.

6 En el campo de la Educación Matemática se presenta un gran volumen de investigaciones que tratan las similitudes y diferencias entre tales abordajes, no se recuperan en este artículo por limitaciones de espacio.
} 
se pone en escena la necesidad de que futuros profesores se apropien de conocimientos necesarios para llevar a cabo actividades de modelado.

Con la propuesta buscamos crear experiencias matemática y didácticamente ricas y que los sujetos las interpreten después de vivirlas, considerando que las personas principalmente piensan y aprenden a través de experiencias que atraviesan (Gee, 2008). Este autor afirma que interpretar la experiencia significa pensar en la acción antes y después de ejecutarla, analizar cómo los objetivos se relacionan con el propio razonamiento de la situación, extraer las lecciones aprendidas y anticipar cuándo y dónde podrían ser útiles. En esta misma línea Dewey (1958) afirma que la educación debe basarse en la experiencia, y más aún, Larrosa (2003) señala que a partir de la experiencia es posible dar sentido a lo sucedido.

Bajo los supuestos descriptos, en este artículo tenemos como objetivos describir, analizar y discutir una experiencia de formación para futuros profesores en un contexto de MM vinculada con fenómenos geométricos. Además avanzamos en la presentación de resultados relevantes destacando producciones emergentes del trabajo realizado por estudiantes que cursaron GEE en el año 2018 en la UNL.

Con el fin de dar cuenta de los objetivos planteados en el siguiente apartado presentamos los constructos teóricos en los que se basa la propuesta, posteriormente la describimos y presentamos producciones de los futuros profesores. Finalmente reflexionamos en torno al sentido de la experiencia desarrollada.

\section{CONSTRUCTOS TEÓRICOS QUE APOYAN EL DISEÑO DE LA PROPUESTA}

El diseño de la propuesta de enseñanza toma aportes e ideas vinculadas con la MM como abordaje pedagógico. Bassanezi (1994) afirma que emplear la MM como abordaje de enseñanza y medio para el aprendizaje posibilita el desarrollo de ciertos modos de pensamiento y actuación, entre otros, la producción de conocimientos, la realización de abstracciones y formalizaciones y el establecimiento de generalizaciones y analogías. Es importante destacar que bajo este abordaje se pretende que los estudiantes no solo puedan aplicar conocimientos matemáticos ya conocidos, sino que emerjan nuevos, es decir, se produzcan conocimientos que se irán configurando o reconfigurando en el proceso de MM.

La perspectiva mencionada se sustenta en una visión de la matemática como actividad cuasi-empírica (Lakatos, 1978; Ravn y Skovsmose, 2019). La adopción de esta perspectiva supone asumir que la exploración por parte de los 
estudiantes constituye un aspecto central de la enseñanza, así como la posibilidad de proporcionar oportunidades para que éstos apelen al sentido común y a sus experiencias (Sriraman y Mousoulides, 2014). En este sentido, Lakatos (1978) afirma que:

Las matemáticas informales y cuasi-empíricas no se desarrollan mediante un monótono aumento del número de teoremas indubitablemente establecidos, sino que lo hacen mediante la incesante mejora de las conjeturas, gracias a la especulación y a la crítica, siguiendo la lógica de pruebas y refutaciones. (p.20).

En relación con lo mencionado, en la figura 1 se muestra un esquema de MM (Bassanezi, 2002). Si bien este esquema se focaliza en el proceso de MM en el ámbito matemático, el mismo se puede emplear como inspiración para pensar la MM como abordaje pedagógico (Esteley, 2014).

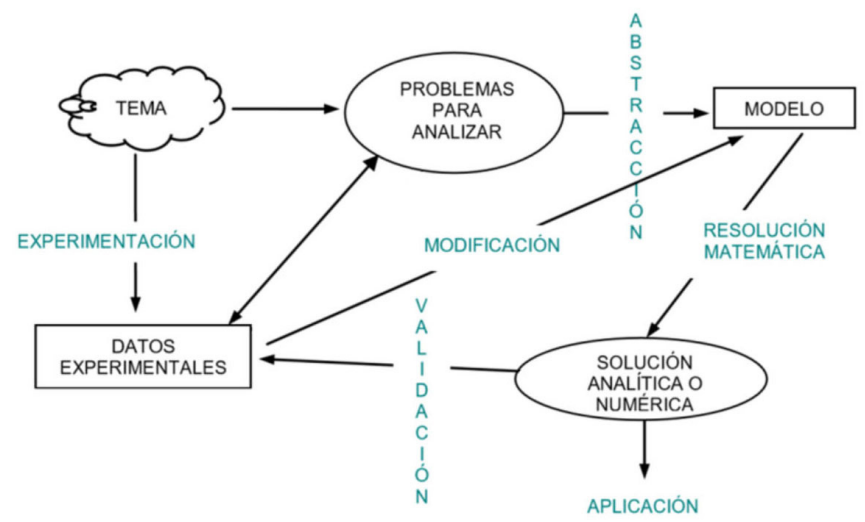

Figura 1: Esquema de proceso de modelización matemática.

Acorde a dicho esquema, quienes se involucran con la MM formulan problemas en el marco de un tema en estudio, emplean datos existentes o producen nuevos con el fin de construir un modelo que dé respuestas al problema, se valida el modelo y en caso necesario se modifica. En ese sentido, el proceso es cíclico e incluye construcciones provisorias.

Cabe notar que el modelo provisorio o final puede tomar diversas formas: analítica, gráfico, verbal, entre otros (Esteley, 2014). Este proceso, como afirma la autora, permite que el o los modelos se modifiquen o convaliden en función de 
los resultados alcanzados en cada momento del proceso. Esta característica de la MM como abordaje pedagógico resulta eficiente para fomentar la construcción de conocimiento.

Entendemos como modelo la definición aportada por Zahar (1989, p.170): "Modo de explicación, construcción teórica, idealizada, hipotética, que sirve para el análisis o evaluación de una realidad concreta". Bassanezi (1994) pone de manifiesto que el trabajo con MM puede tener importancia intrínseca independientemente de su aplicación a la vida diaria. En el modelado el comienzo es simplemente un problema en el que aún se desconoce la matemática que se pondrá en juego. En esta misma línea, Davis y Hersh (1989) consideran los modelos como herramientas desarrolladas por el hombre para proveer una descripción viable de la realidad social, de la naturaleza o de la propia matemática, cumpliendo una doble función: medio para pasar información y dispositivo para pensar. En concordancia con la postura de Bassanezi $(1994$; 2002) se hace referencia a modelos producidos libremente por estudiantes, emergentes de su propia construcción y que se consideran intermediarios mediante los cuales se idealiza o simplifica una realidad o teoría compleja para acceder a un tratamiento matemático más formal.

Si bien en general, en el trabajo de Bassanezi (1994; 2002) se privilegian temas y problemas de la realidad perceptual, Freudenthal (1991) guardando coherencia con los principios del trabajo de MM, sugiere ampliar los fenómenos a estudiar. Y no se limita a pensar en el estudio de fenómenos del mundo real (perceptual), sino que resalta la importancia de que los estudiantes enfrenten situaciones que tienen la capacidad de visualizar o imaginar por la influencia de experiencias previas.

En la propuesta de enseñanza que se presenta, el docente cumple un rol de guía y organizador de las interacciones, es el mediador entre las producciones de los estudiantes y la matemática reconocida por la comunidad matemática (Freudenthal, 1991). De este modo, se evidencia el carácter social de la matemática, donde la reflexión colectiva redunda en el avance hacia niveles de comprensión cada vez más altos respecto a la matemática en juego.

En instancias de observación y estudio de la propuesta se busca poner atención en los procesos de validación en el marco de los procesos de MM seguidos. Bassanezi (2002) considera que la misma se presenta cuando se compara el modelo matemático con datos reales o información disponible, de modo tal de posibilitar la aceptación o el rechazo del modelo. A su vez, señala que el grado de aproximación deseado es un factor predominante en la decisión 
de aceptar o rechazar el modelo y esto permite la reconfiguración de conocimientos. En este sentido, éstos pueden asumir un carácter provisorio.

Balacheff (2000) realiza una investigación en la que estudia procesos de pruebas puestos en juego por estudiantes, y se refiere a la validación en el ámbito de trabajo matemático:

En este estudio utilizaremos la palabra razonamiento para designar la actividad intelectual no completamente explícita que se ocupa de la manipulación de la información dada o adquirida, para producir una nueva información. Le asignaremos el término procesos de validación a esta misma actividad cuando tenga como fin asegurarse de la validez de una proposición y, eventualmente, producir una explicación (una prueba o una demostración). (p.13)

Para este trabajo tomamos como referencia el sentido de validación presente en Bassanezi (2002) o Balacheff (2000). Estas ideas abren la posibilidad de una reflexión colectiva entre estudiantes que permita un avance hacia niveles de comprensión cada vez más altos y quizás más formales que habiliten luego una prueba.

\section{LA PROPUESTA DE ENSEÑANZA: UNA MIRADA HACIA EL DISEÑO Y HACIA LAS PRODUCCIONES}

\subsection{Consideraciones GeNeRALES PARA EL DISEÑO}

El programa de formación docente en el que llevamos adelante la propuesta tiene una duración de 5 años. Las asignaturas se organizan de modo tal que pueden agruparse en cuatro categorías principales: matemáticas específicas, educación general, educación matemática y formación general. La asignatura GEE pertenece a la primera categoría, se dicta en el primer semestre del tercer año y los estudiantes inscriptos en ella, previamente, no han cursado asignaturas de educación matemática. Cabe indicar que tanto los estudiantes (Cruz, Esteley y Scaglia, 2018) como las docentes que llevan adelante la propuesta no han experimentado escenarios educativos en los que se emplea la MM.

La propuesta se desarrolló en 17 horas reloj, organizadas en 5 clases de 3 horas cada una y una de 2 horas, durante las tres primeras semanas de cursado. 
Participan de esta experiencia 17 futuros profesores, sin embargo es necesario señalar que no todos se encuentran presentes en la totalidad de las clases.

Previo al diseño de la propuesta las investigadoras acuerdan con las docentes a cargo de la asignatura en escoger "poliedros" como tema principal para abordar en el proceso de MM. Dicho tema no había sido desarrollado con anterioridad en la carrera Profesorado en Matemática y, al implementarse esta propuesta en las primeras clases, tampoco se había trabajado en este curso.

Se establecen dos problemas relacionados con el tema señalado. El primero solicita determinar qué figura o figuras (de un conjunto de figuras dadas) es un poliedro y el segundo invita a conjeturar si existe alguna relación entre el número de vértices, de aristas y de caras de un poliedro. De este modo, el avance sobre el primer problema permite adentrarse en el segundo con un trabajo previo de reconocimiento de variables pertinentes para el estudio de un poliedro. Finalmente, se trabaja con nuevas problemáticas que formulan los estudiantes con el fin de lograr la puesta en juego de un proceso de modelización completo, dado que los estudiantes en interacción con las docentes delimitan libremente un problema a modelizar y buscan los medios para hacerlo (Esteley, 2014).

En la propuesta se invita a la reflexión constante respecto a modelización y a validación en el ámbito de trabajo matemático. Atendiendo las particularidades que implica la formación de futuros profesores en matemática, se buscó favorecer reflexiones vinculadas a la producción matemática y sus vínculos con cuestiones didácticas.

Pensamos los procesos de enseñanza y de aprendizaje como un espacio en el que emergen conocimientos, por lo que posicionamos la clase como comunidad de práctica (estudiantes y docente). En las comunidades de práctica se negocian significados entre los participantes, se trabaja en conjunto hacia objetivos comunes y el aprendizaje puede ser reconocido por cambios en las identidades personales de los miembros de la comunidad (Forman, 2014).

Organizamos la propuesta en siete momentos, dado que asumimos que la construcción de conocimientos es progresiva. La modalidad de trabajo durante la puesta en juego de la propuesta es generalmente en grupos de tres a cinco integrantes, puesto que consideramos que el intercambio entre pares permite confrontar, comparar, contrastar, unificar y consolidar ideas de los sujetos involucrados respecto a las nociones y conocimientos matemáticos que se ponen en juego en las consignas, de modo que logren profundizar y repensar las ideas que emergen durante el trabajo matemático. 
En varios momentos proponemos debate colectivo, dado que consideramos que en escenarios educativos en los que estudiantes presentan al final resultados de un problema en estudio a la comunidad de práctica en la que estén inmersos, tienen un alto valor formativo, pues posibilitan sugerencias para su mejora, intercambios de experiencias y críticas, mejoras de cada producción, entre otros. En definitiva, propician la actividad de comunicar ideas matemáticas y contribuir con una producción de conocimientos.

Finalmente señalamos que durante la puesta en juego de la propuesta sugerimos que los estudiantes completen las consignas en Google Drive compartido por la comunidad de práctica de modo tal de asegurar en todo momento el acceso a la producción de todos los grupos durante la clase. En caso que no se disponga de conexión a internet sugerimos que las producciones se realicen en Word con el fin de que luego las investigadoras y docente del curso recuperen, organicen y compartan las producciones.

\subsection{LA PROPUESTA DE ENSEÑANZA}

Organizamos la propuesta en siete momentos. En los momentos uno y cinco se reflexionan y abordan cuestiones en torno a producción matemática y sus enlaces con la didáctica, particularmente se pone énfasis en discusiones que versan en la noción de MM y validación. En los momentos dos, tres y cuatro se trabaja en torno al primer problema matemático, determinar qué figura o figuras (de un conjunto de figuras dadas) es un poliedro. En el momento seis, se aborda el segundo problema matemático, conjeturar si existe relación entre el número de vértices, de aristas y de caras de un poliedro y qué característica tiene. Finalmente, en el momento siete, se propone la formulación, resolución y presentación de un problema geométrico por parte de los futuros profesores.

A continuación presentamos un esquema (Figura 2) en el que caracterizamos sintéticamente la propuesta. 


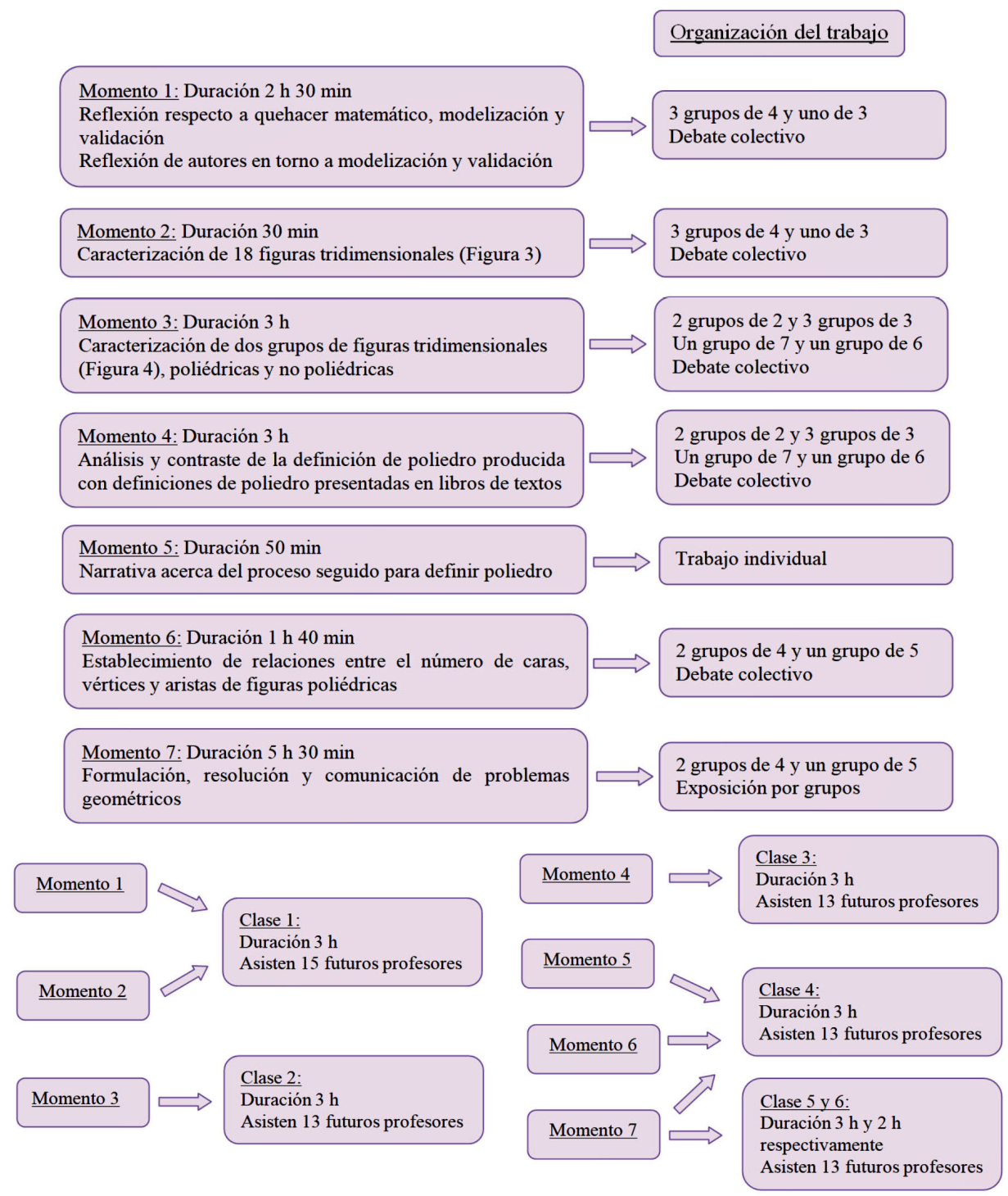

Figura 2. Síntesis de la propuesta. 
A continuación presentamos detalles de cada momento, explicitamos las consignas presentadas a los estudiantes, el modo de trabajo y avanzamos presentando algunas producciones del trabajo de los futuros profesores que participan de la experiencia.

\subsubsection{Momento 1}

El primer momento se lleva adelante en la primera clase de la asignatura GEE a la que asisten 15 futuros profesores que se organizan en cuatro grupos, 3 de 4 estudiantes y uno de 3. La consigna que se presenta es la siguiente:

Lean en grupos los siguientes interrogantes y discutan posibles respuestas a los mismos. Luego realicen una narración en la que recuperen las respuestas de todos los integrantes del grupo (explicitando los acuerdos y desacuerdos que hayan surgido durante las discusiones): a- ¿Cuáles son las actividades que lleva a cabo una persona que hace Matemática? b- ¿Qué entienden por proceso de validación en instancias del trabajo matemático? c- ¿Qué entienden por proceso de modelización matemática?

Esta instancia de trabajo permite poner de manifiesto el sentido otorgado al quehacer matemático, procesos de validación y de modelización a partir de experiencias educativas previas, atendiendo a que las palabras producen sentido (Larrosa, 2003).

Posteriormente presentamos a cada grupo (organizados del mismo modo que en la instancia anterior) un breve escrito que incluye el esquema de modelización ilustrado en la Figura 1, un texto en que se expone, siguiendo a Esteley (2014), el modo en que se utilizan algunos términos presentes en el esquema (experimentación, abstracción, resolución, validación, modificación y aplicación) y lo propuesto por Balacheff (2000) acerca de proceso de validación. Buscamos que los estudiantes logren interpretar y analizar constructos teóricos respecto a procesos de validación y de modelización.

Las consignas presentadas son las siguientes: a- A continuación se les presenta uno de los posibles esquemas en el que se muestra un proceso de modelización (cuyo autor es Bassanezi) y se explicita el modo en que se utilizan algunos términos presentes en el esquema. Interpreten lo presentado a fin de responder qué es o cómo se puede entender un proceso de modelización. b- Además de considerar la visión de validación propuesta por Bassanezi, se presenta la de Balacheff (2000) acerca de proceso de validación. A partir de los 
aportes de estos dos autores formulen una idea sobre qué es o como se puede entender un proceso de validación en el ámbito del trabajo matemático.

Luego de esta etapa proponemos una discusión colectiva en la que cada grupo pone de manifiesto la producción realizada. Los futuros profesores exponen a la comunidad de práctica la respuesta realizada inicialmente, el análisis de los autores y contrastan su producción con la interpretación realizada a partir de la lectura de los autores.

\subsubsection{Momento 2}

El segundo momento también se lleva adelante en la primera clase y aquí se da inicio al trabajo de MM. Como se menciona anteriormente, el primer problema matemático que proponemos es determinar qué figura de un grupo de figuras dadas representa un poliedro. Esto es, los estudiantes deberán construir herramientas o instrumentos conceptuales que les permitan discernir acerca de si cada figura es o no un poliedro.

Para iniciar el trabajo en torno a este problema matemático comenzamos solicitando lo siguiente: Observen las siguientes imágenes (ver figura 3) con el fin de determinar características que poseen.

El modo de trabajo se propone en grupos organizados del mismo modo que en el momento anterior. La idea en esta instancia es que los futuros profesores caractericen, apelando a variables escogidas por ellos, figuras tridimensionales. Entregamos a cada estudiante una impresión con las imágenes y se encuentran disponibles en el aula representaciones en material concreto de las mismas. Algunas de tales imágenes representan objetos de la realidad y otras son construcciones realizadas con el Software de Geometría Dinámica GeoGebra. Entre las imágenes se presentan ejemplos y no ejemplos de poliedros. Consideramos como no ejemplos, tomando aportes de Tall (1989) a las representaciones que no cumplen las condiciones de una definición formal de poliedro, y a las que cumplen con algunas condiciones y no con otras, como se muestra en la figura 3. 


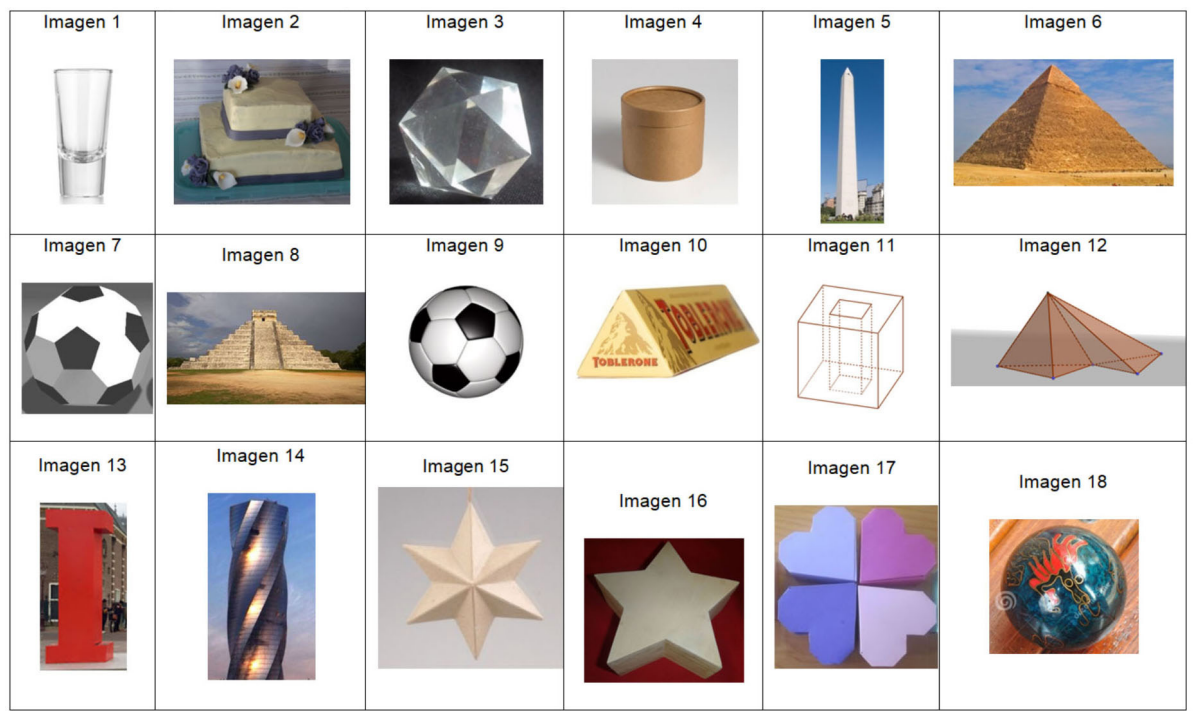

Figura 3. Imágenes presentadas a los estudiantes.

Cabe destacar que en el primer momento dejamos libertad de elección, dado que los estudiantes determinan las características. En este sentido es amplio el abanico de oportunidades de elección: colores, texturas, formas de caras, etc. Sin embargo, en instancias de resolución predomina la selección de características geométricas, posiblemente esto se debe a que se encuentran en un curso de geometría y son estudiantes avanzados en una carrera de profesorado en matemática. Finalmente proponemos una instancia de debate colectivo donde cada grupo expone a la comunidad de práctica el trabajo realizado.

\subsubsection{Momento 3}

El momento tres de la propuesta se lleva adelante en la segunda clase. En la misma se encuentran presentes 13 estudiantes. En este momento presentamos las mismas imágenes que en la clase anterior, pero organizadas en dos grupos (Figura 4), las que consideramos no poliédricas (Grupo 1) y las poliédricas (Grupo 2). 

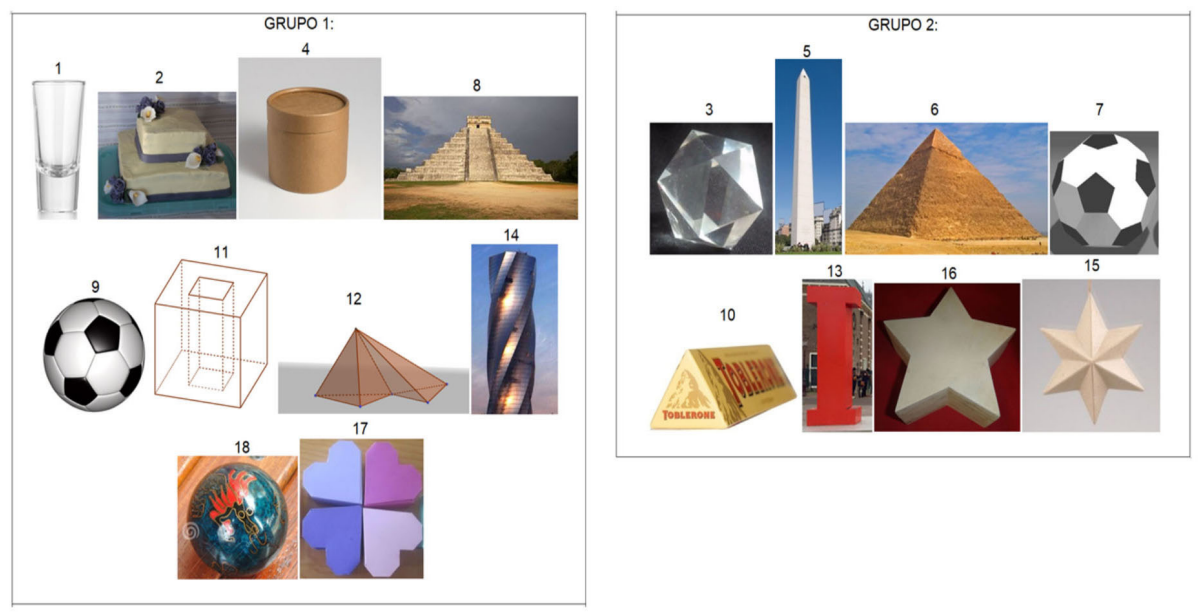

Figura 4. Imágenes organizadas en dos grupos.

En una primera instancia trabajan en cinco grupos, dos grupos de dos estudiantes y tres grupos de tres. Las consignas que presentamos son las siguientes:

a) Las imágenes presentadas en la clase anterior se organizan en dos grupos. Determinen características geométricas que permitan que se agrupen de ese modo. ¿Qué nombre le podrías al grupo 1?, y, ¿al grupo 2?

b) Representen en el Software de Geometría Dinámica GeoGebra una figura tridimensional que pertenezca al grupo 1 y una que pertenezca al grupo 2 (diferentes a las presentadas anteriormente). Expliquen por qué consideran que dichas representaciones pertenecen respectivamente a los grupos 1 y 2 .

En una segunda instancia, proponemos que se junten con otro grupo y respondan la siguiente consigna: Discutan con otro grupo las respuestas de los incisos anteriores. Establezcan una única caracterización y determinen dos representaciones en el Software de Geometría Dinámica GeoGebra, una que pertenezca a cada grupo.

Los estudiantes se organizan en 2 grupos, uno de seis estudiantes y uno de siete. En este momento se busca que los estudiantes logren reconocer características de figuras poliédricas y no poliédricas, validar sus decisiones, 
iniciar el trabajo de construcción del concepto figural de poliedro (Fischbein, 1993), iniciar la construcción de figuras geométricas con GeoGebra en concordancia con las características propuestas, reconocer la equivalencia entre definiciones, entre otros.

En las tablas 1 y 2 exponemos las producciones emergentes de esta instancia. Cabe indicar que las trascripciones de lo escrito por los estudiantes son textuales y aunque puedan presentar algún error o inconveniente las transcribimos sin modificación. En la tabla 1 explicitamos la producción del grupo formado por siete futuros profesores y en la 2 la del grupo de seis.

Tabla 1. Producción de estudiantes del grupo 1 referidas al segundo momento.

\begin{tabular}{|l|l|c|}
\hline \multicolumn{2}{|c|}{ Características determinadas } & Representación realizada en el SGD \\
\hline Giguras 1 & $\begin{array}{l}\text { No todos tienen vértices, caras o } \\
\text { aristas, no todas sus caras son po- } \\
\text { lígonos, algunos tienen huecos. } \\
\begin{array}{l}\text { Elegimos como figura dos conos } \\
\text { invertidos con sus vértices en } \\
\text { común. }\end{array}\end{array}$ \\
\hline Figuras \\
Grupo 2
\end{tabular}


Tabla 2. Producción de estudiantes del grupo 2 referidas al segundo momento.

\begin{tabular}{|c|c|c|}
\hline \multicolumn{2}{|r|}{ Características determinadas } & Representación realizada en el SGD \\
\hline $\begin{array}{l}\text { Figuras } \\
\text { Grupo } 1\end{array}$ & $\begin{array}{l}\text { Notamos dos grandes grupos entre estos } \\
\text { grupos los cuales son: los cuerpos que } \\
\text { ruedan y los que tienen base. Además, } \\
\text { hay cuerpos que son huecos y que son } \\
\text { sólidos. }\end{array}$ & 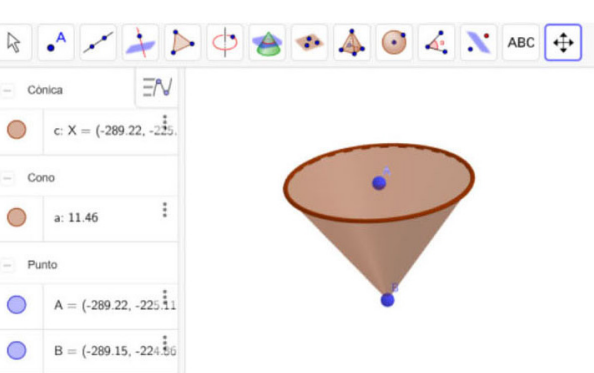 \\
\hline $\begin{array}{l}\text { Figuras } \\
\text { Grupo } 2\end{array}$ & $\begin{array}{l}\text { Figuras poliédricas: } \\
\text { Las figuras dadas son superficies poliédri- } \\
\text { cas, es decir, un conjunto finito de polígo- } \\
\text { nos que tienen las siguientes características: } \\
\text { Cada lado de una cara es compartido } \\
\text { con otra y solo otra. } \\
\text { Las caras contiguas están en distinto plano. } \\
\text { Convexas: Icosaedro, dodecaedro, pirámi- } \\
\text { de, obelisco y toblerone. } \\
\text { No convexas: Las estrellas y la "i" } \\
\text { Regulares: El icosaedro y el dodecaedro. } \\
\text { No regulares: Los restantes. }\end{array}$ & 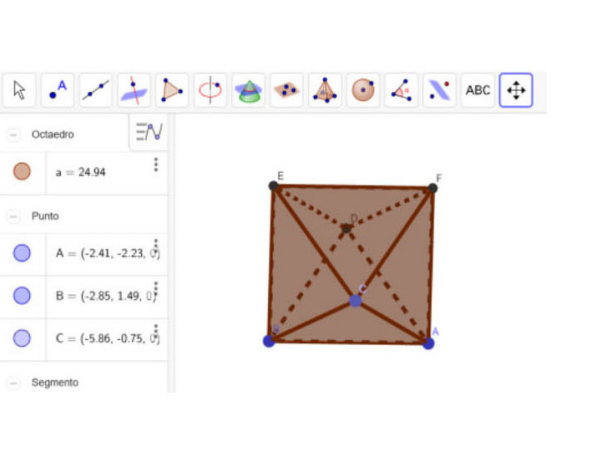 \\
\hline
\end{tabular}

En las producciones, desarrolladas mediante el uso de variables pertinentes, vínculos relevantes o categorizaciones, se presenta una primera aproximación a la definición de poliedro, que permite explicar, construir teóricamente e idealizar la noción que posibilitaría luego el análisis de figuras para determinar o discernir si una figura es o no poliedro.

En este sentido, cabe destacar que "En algunos casos son construidos modelos, cuando la situación original es muy compleja para ser estudiada. Se espera que el modelo traiga algún esclarecimiento o información sobre la situación más compleja" (Gazzetta, 1989, p.18) y de ese modo avanzar en la búsqueda de al menos explicaciones provisorias. En este caso, las caracterizaciones de las figuras permiten identificar condiciones que, a su vez, conducirán a la elaboración de definiciones. 
Las descripciones y caracterizaciones se constituyen en un primer modelo emergente del trabajo con MM (Cruz, Scaglia y Esteley, 2018; Cruz, Scaglia y Esteley, 2019 b), lo que brinda la oportunidad a los estudiantes de iniciar el camino para producir modelos más complejos, o en este caso particular una definición más clara o más compleja que luego puede ser asumida por la comunidad de práctica.

Respecto al empleo de GeoGebra, cabe destacar que es la primera vez que los estudiantes del grupo 1 emplean su vista 3D, en el grupo 2 algunos de los estudiantes ya cursaron GEE por lo que conocen esta herramienta. Ambos grupos lograron realizar las dos construcciones, descubrir comandos y modos de emplear este software, por ejemplo el uso de vistas 2D y 3D en simultáneo. A la vez, resignifican conceptos geométricos que habían trabajado en un curso de geometría plana, previo a la asignatura GEE, como el de simetría central (ver en tabla 1 los conos simétricos respecto del vértice). Estas últimas cuestiones son propias de procesos de $\mathrm{MM}$, donde emergen conocimientos matemáticos y tecnológicos no previstos o que no se sabe que surgirán cuando se inicia el mismo (Bassanezi, 1994).

En instancias de discusión colectiva, con aportes de todos los estudiantes que se encuentran presentes en la clase y docentes, producen la definición que se muestra en la tabla 3:

Tabla 3. Definición de poliedro construida en la comunidad de práctica y transcripción del texto.

\begin{tabular}{|c|c|}
\hline 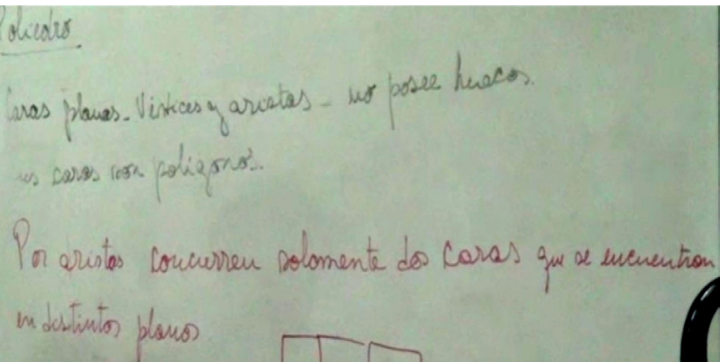 & $\begin{array}{l}\text { Transcripción de lo escrito en el pizarrón: } \\
\text { Poliedro } \\
\text { Caras planas- Vértices y aristas - No } \\
\text { posee huecos. } \\
\text { Sus caras son polígonos. } \\
\text { Por aristas concurren solamente dos } \\
\text { caras que se encuentran en distintos } \\
\text { planos. }\end{array}$ \\
\hline
\end{tabular}




\subsubsection{Momento 4}

El momento tres concluye con la producción del primer modelo (tabla 3 - definición provisoria de poliedro) de la comunidad de práctica. Continuamos la propuesta buscando poner a prueba la producción, incentivando la validación y la decisión por parte de los futuros profesores acerca de la modificación o no del modelo.

Este momento se lleva a cabo en la tercera clase. Asisten 13 estudiantes y se organizan en tres grupos, dos de cuatro y uno de cinco. Las consignas presentadas son las siguientes:

a) Busquen por lo menos 2 definiciones de poliedros. La búsqueda la pueden realizar en libros de texto, libros de geometría (reconocidos por la comunidad matemática), internet, entre otros.

b) Decidan y expliquen si las definiciones buscadas recientemente son equivalentes. De las imágenes presentadas en la consigna anterior, cexisten figuras tridimensionales que cumplen alguna de las definiciones y no otras?

c) Justifiquen si las definiciones buscadas son o no equivalentes a la establecida en la comunidad matemática "clase de geometría". De las imágenes presentadas en la consigna anterior, ¿existen figuras tridimensionales que cumplen alguna de las definiciones y no otras?

d) Preparen una presentación para realizar al resto de la clase, en la que se exponga lo pensado en los incisos anteriores. Tengan en cuenta explicitar y argumentar si la definición establecida en la comunidad matemática "clase de geometría" les sigue pareciendo adecuada.

Luego del trabajo grupal proponemos que se lleve adelante un debate colectivo. En el mismo, cada grupo expone el trabajo realizado. Para cerrar este momento, las docentes a cargo del curso deciden por restricciones de tiempo, establecer y delimitar la definición (formulada por las docentes tomando aportes de Puig Adam (1980)) que se empleará en las clases de GEE y se utilizará como modelo en el desarrollo de esta asignatura para validar acciones futuras. Cabe señalar que la decisión de finalizar este momento con una definición formulada por el cuerpo docente, sin reconsiderar las ideas de los estudiantes 
no es una decisión consensuada previamente con las investigadoras. Si bien se entiende que esta decisión es tomada quizás por la necesidad de cumplir con el programa de la asignatura, a partir de esta acción docente consideramos que se produjo un truncamiento del proceso de $\mathrm{MM}$ al establecer una definición sin tener en cuenta las voces y producciones de los futuros profesores. No obstante, la riqueza del trabajo de los estudiantes no queda oculta y serán consideradas en ámbitos investigativos.

\subsubsection{Momento 5}

El momento cinco se lleva adelante en la cuarta clase, en la misma se encuentran presentes 13 estudiantes. En este momento se busca cerrar el trabajo matemático en torno al primer problema, por lo que invitamos a los futuros profesores a realizar una narración escrita individual. La consigna presentada es la siguiente: Tomando como referencia el esquema del proceso de modelización presentado en la clase 1 describan el proceso seguido al interior de la comunidad para arribar a una definición o modelo de la noción de "poliedro". Dentro del proceso de modelización, ¿cómo vivieron la validación?

Esta instancia es fundamental porque permite a cada partícipe de la experiencia reflexionar y recuperar individualmente lo vivido en instancias de una experiencia colectiva, pues cada sujeto reconstruye los hechos atendiendo al modo en que atravesó y lo atravesó la experiencia, produciendo así sentido a la vivencia (Larrosa, 2003).

\subsubsection{Momento 6}

En el momento seis se lleva adelante el trabajo de MM en torno al segundo problema matemático, conjeturar si existe relación entre el número de vértices, de aristas y de caras de un poliedro tomando como referencia empírica las figuras que determinamos como poliédricas (grupo 2) de la figura 4. Detrás de este problema se hace evidente la relación de Euler. Este momento, al igual que el momento cinco, se ejecuta en la cuarta clase. Para esta instancia proponemos que se organicen en 3 grupos, dos de cuatro y uno de cinco.

Comenzamos explicitando la siguiente consigna: 
a) Cuando trabajaron con polígonos planos pudieron observar (o no) que el número de aristas coincide con el número de vértices. Por ejemplo, el cuadrado tiene cuatro aristas y cuatro vértices, el pentágono cinco aristas y cinto vértices, etc. Cabe preguntarse entonces, para los poliedros, ¿será posible establecer relaciones entre los números de caras, de vértices y de aristas de los mismos? Los invitamos a que levanten o traten de levantar algunas conjeturas al respecto (decir que no o si es también una conjetura).

b) En caso de haber encontrado alguna relación representen en el software de geometría dinámica GeoGebra figuras tridimensionales que la cumplen y en la hoja de cálculo expliciten la relación.

Cabe indicar que en el grupo 2 de figuras tridimensionales presentado en la figura 3, los "poliedros" cumplen la relación de Euler. Sería una discusión teórica y epistemológica interesante el analizar si es necesario que todo poliedro cumpla la relación de Euler o no, pero no abordaremos ese punto en este artículo.

En el trabajo en aula, llevado a cabo en 3 grupos de 4 estudiantes cada uno, surgen las producciones que fueron escritas en la pizarra y que se muestran en la tabla 4.

Tabla 4. Pizarra de la puesta en común realizada en el momento 6 y transcripción del texto.

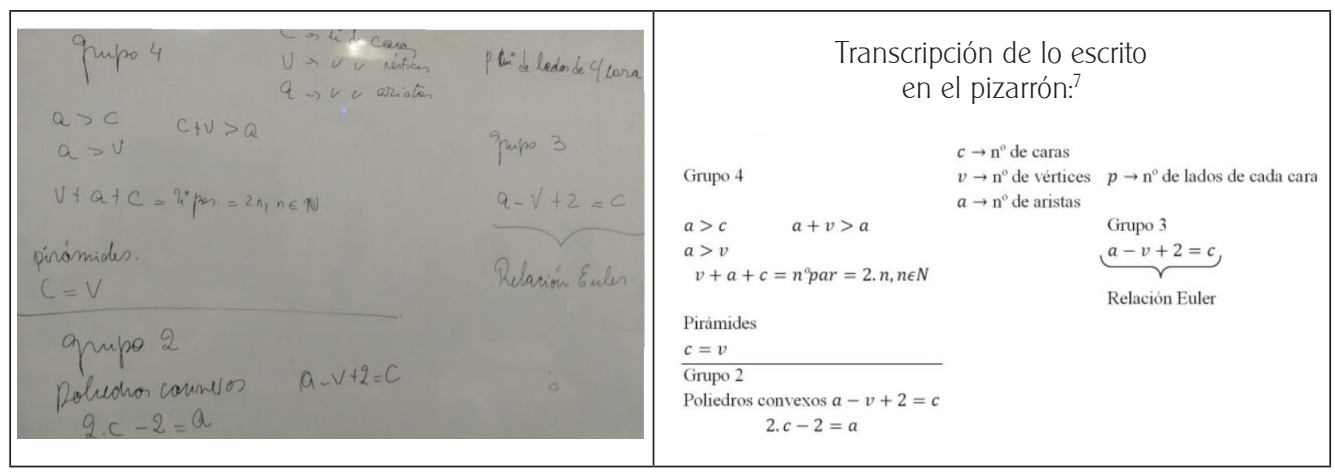

${ }^{7}$ El nominar 2, 3 y 4 a los grupos se debe a que desde la primera clase trabajan de ese modo. Los estudiantes del grupo 1 no mantienen la formación del mismo por ausentarse a diversas clases. 
Apreciamos que el grupo 4 encuentra diversas relaciones estableciendo hipótesis para limitarlas para ciertos tipos de poliedros. El grupo 2 determina una fórmula para poliedros convexos y luego la relación de Euler para poliedros. La fórmula no se verifica para todo poliedro convexo, sin embargo los estudiantes que realizan esta conjetura afirman que es válida para los poliedros convexos en los que contaron.

El grupo 3 formado por estudiantes que habían cursado previamente la materia y estudiantes que se enfrentan por primera vez a la asignatura arriban a la fórmula de Euler. En este grupo, una de las estudiantes recordaba la relación de Euler y manifiesta que no va a opinar para dar espacio a la formulación de conjeturas por sus compañeras.

Posteriormente en instancias de trabajo colectivo surgen interesantes discusiones respecto a la posibilidad de validación de las conjeturas establecidas.

\subsubsection{Momento 7}

Organizamos el momento siete en tres instancias: la formulación, resolución y exposición de un problema geométrico formulado por los futuros profesores. La formulación de problemas se lleva adelante en la clase cuatro, la resolución en la clase cinco y la exposición de la resolución en la clase seis. En estas clases asisten 13 estudiantes que se organizan para trabajar en dos grupos de cuatro y un grupo de cinco.

La consigna presentada con el fin de solicitar la formulación de problemas geométricos es la siguiente: Durante las clases de Geometría Euclídea Espacial Ilevadas a cabo hasta el momento pensaron y reflexionaron diferentes problemas, por ejemplo, la definición de poliedros, relaciones entre números de caras, vértices y aristas en poliedros, entre otros. Ahora los invitamos a que se hagan preguntas que les surgen a partir del proceso vivido con el fin de que ustedes mismos determinen un problema geométrico.

Los problemas geométricos diseñados por ellos se presentan en la tabla 5. 
Tabla 5. Problemas planteados en términos de interrogantes por los tres grupos.

\section{GRUPO 2:}

Nosotras pensamos que si tenemos distintas figuras geométricas (triángulos, rectángulos, cuadrados, pentágonos, etc.) al unirlos podemos formar poliedros. Pero ¿isiempre se puede? (tomando cualquier figura y cantidad).

Nuestra idea es ver que la respuesta va a depender de distintas características de las figuras y lo analizaremos en la clase con los demás grupos utilizando el Polydron y otros elementos (figuras regulares, irregulares, circulares).

GRUPO 3:

Con las características descriptas en los poliedros, el concepto de ángulo no se menciona. Dado los contenidos estudiados en geometría plana acerca de polígonos, nos preguntamos si existen o no ángulos en el espacio y como se definen, miden y clasifican, si existen ángulos exteriores.

GRUPO 4:

Durante estas clases el problema que nos surgió es que en las definiciones aparecen términos que no comprendíamos, por ejemplo: superficie y sólido y esto nos dificultó poder comprenderlos. ${ }^{8}$

De los problemas formulados por los grupos apreciamos que todos eligen trabajar con situaciones de la geometría tridimensional, a pesar de que la consigna es abierta a un problema geométrico cualquiera.

Luego de este momento en la clase cinco proponemos lo siguiente:

a) Intenten junto a los compañeros con los que diseñaron la problemática determinar una posible "reflexión" y "respuesta" a la misma. Preparen para entregar en el próximo encuentro un archivo de Word en el que se expliciten de modo detallado sus exploraciones, investigaciones, conjeturas, conclusiones, entre otros. Se sugiere que finalmente presenten una reflexión en la que narren como vivieron el proceso de producción y exploración de su problemática.

b) Diseñen una presentación de 15 minutos para que en el próximo encuentro puedan mostrar a la comunidad "Clase de geometría" el proceso llevado a cabo al interior del grupo.

${ }^{8}$ La resolución de este problema consiste en la realización de un análisis terminológico para comprender las definiciones. 
A partir de este momento los estudiantes han completado la experiencia en torno al proceso de MM (Esteley, 2014). La resolución de una problemática formulada por los mismos estudiantes da lugar a responder preguntas que ellos mismos se hicieron. Se destaca esta cuestión, dado que es posible que no hayan tenido previamente en su formación una experiencia con estas características. Así mismo, el momento de presentación de la resolución de la problemática permite a los estudiantes comunicar resultados de su propia investigación matemática y poner a prueba su producción frente a compañeros y docentes.

\section{RELFEXIONES FINALES}

Lo descripto ha permitido delinear con detalles una experiencia educativa para futuros profesores. Hacemos evidentes las consignas diseñadas apelando a MM como abordaje pedagógico. Ofrecemos evidencias o ejemplos de las producciones que emergen en el trabajo en aula, así como también posibilidades de los estudiantes para comprometerse con un trabajo de MM y producir conocimientos matemáticos y tecnológicos en esas instancias.

Los futuros profesores que participan en esta experiencia se apropian del proceso de MM de naturaleza intra-matemático. En este sentido, cabe señalar que trabajan con referentes empíricos vinculados con la matemática y recurren a conocimientos ya apropiados de naturaleza matemática para construir modeIos, como las definiciones de poliedro, en el sentido de Gazzetta (1989), en una primera instancia o las formulaciones de relaciones al interior de figuras que luego validan recurriendo a diversos procedimientos.

Esta experiencia resulta valiosa para los propios estudiantes y para las docentes del curso. Se han dado evidencias de un trabajo con MM centrado en objetos matemáticos, que da cuenta de las posibilidades formativas que estos procesos de producción de conocimientos otorgan a futuros profesores. Así mismo, la reflexión de los procesos de MM y validación posibilita que emerjan conocimientos didácticos, matemáticos y tecnológicos.

Lo discutido desde una mirada pedagógica está en proceso de análisis en el ámbito investigativo. Así mismo, señalamos la importancia que ha tenido en esta experiencia el trabajo en conjunto entre investigadores y docentes a fin de contribuir en la formación de futuros profesores en matemática. En el ámbito de investigación se logra dilucidar las ideas iniciales de los estudiantes acerca de quehacer matemático, validación y modelización (Esteley, Cruz y 
Scaglia, 2019; Cruz, Scaglia y Esteley, 2019 a), este avance pone en evidencia la importancia del vínculo entre investigadores y docentes. Este vínculo se encuentra en estrecha relación con la recomendación propuesta por Ouvrier-Buffet (2015) respecto a la necesidad de involucrar a profesores universitarios de matemáticas e investigadores en educación matemática, a fin de implementar actividades de producción de definiciones a nivel universitario.

Las características de la propuesta diseñada por las investigadoras, que invita a la construcción de una definición de poliedro, finalmente aporta a la asignatura GEE. Esto es así pues en el material de cátedra empleado en la asignatura, en un principio no se explicitaba la definición de poliedro. A partir de la propuesta de esta experiencia las docentes formulan una definición de poliedro que luego presentan a todo el curso.

Finalmente es de destacar que, todo este trabajo, no hubiese sido posible sin el compromiso asumido por los futuros profesores que cursaban GEE en 2018. Para ellos como así también para los docentes del curso formulamos un especial agradecimiento.

\section{REFERENCIAS}

Adam, P. (1980). Curso de Geometría Métrica. Euler, G. Puig Ediciones.

Bakker, A y van Eerde, D. (2015). An Introduction to Design-Based Research with an Example From Statistics Education. En A. Bikner-Ahsbahs, C. Knipping y N. Presmeg (Eds.), Approaches to Qualitative Research in Mathematics Education (pp. 429-466). Springer.

Balacheff, N. (2000). Procesos de prueba en los alumnos de matemáticas. Una empresa docente.

Bassanezi, R, (1994). Modelling as a teaching-learning strategy. For the Learning of Matehematics, 14(2), 31-35.

Bassanezi, R. (2002). Ensino-aprendizagem con modelagem matemática: uma nova estratégia. Editora Contexto.

Blum, W. (2015). Quality teaching of mathematical modelling: What do we know, what can we do? In S. Cho (Ed.), The Proceedings of the 12th International Congress on Mathematical Education (pp. 73-96). Springer.

Blum, W., y Niss, M. (1991). Applied Problem Solving, Modelling, Applications and Links to Other Subjects-State Trends and Issues in Mathematics Instruction. Educational Studies in Mathematics, 22, 37-68. 
Borko, H; Dole, S., Esteley, C., Huang, R., Karsenty, R., Miyakawa, T., Da Ponte, P., Potari, D; Robutti, O. y Trouche, L. (2019). Teachers of mathematics working and learning in collaborative groups-discussion document. https://www.mathunion.org/fileadmin/ ICMI/ICMI\%20studies/ICMI\%20Study\%2025/190218\%20ICMI-25_To\%20Distribute_190304_edit.pdf

Confrey, J. (2006). The Evolution of Design Studies as Methodology. En R. Keith Sawyer (Ed.), The Cambridge Handbook of the Learning Sciences (pp. 135-151). Cambridge University Press.

Cruz, M.F., Esteley, C. y Scaglia, S. (2018). Validar, justificar, demostrar en voces de futuros profesores en matemática. En Di Franco (Ed.), VII Reunión Pampeana de Educación Matemática (pp. 105-116). Universidad Nacional de la Pampa.

Cruz, M.F., Scaglia, S. y Esteley, C. (2018). La construcción de definiciones geométricas. En Di Franco (Ed.), VII Reunión Pampeana de Educación Matemática (pp. 42-47). Universidad Nacional de la Pampa.

Cruz, M.F., Scaglia, S. y Esteley, C. (2019 a). ¿Qué piensan futuros profesores de la modelización matemática? En J. Aguirre, L. Proasi y C. De Laurentis (Comp.), Congreso Latinoamericano Prácticas, problemáticas y desafíos contemporáneos de la Universidad y del Nivel Superior. Eje: Prácticas, problemáticas y desafíos contemporáneos de la enseñanza de la Matemática en la Universidad y en el Nivel Superior. (pp. 49-55). Universidad Nacional de Mar del Plata.

Cruz, M.F., Scaglia, S. y Esteley, C. (2019b). Experiencia de modelización matemática con profesores y futuros profesores. http://conferencia.ciaem-redumate.org/index.php/ xvciaem/xv/paper/viewFile/734/439

Davis, P. y Hersh, R. (1989) Experiencia Matemática. Editorial Labor.

Dewey, J. (1958). Experiencia y educación. Losada.

Esteley, C. (2014). Desarrollo profesional en escenarios de modelización matemática: Voces y Sentidos. Filosofía y Humanidades/UNC.

Esteley, C., Cruz, M.F. y Scaglia, S. (2019). Quehacer matemático y validación: ideas de futuros profesores. http://conferencia.ciaem-redumate.org/index.php/xvciaem/xv/ paper/viewFile/729/45

Even R. y Ball D. (Ed.). (2009) The Professional Education and Development of Teachers of Mathematics. Springer.

Fischbein, E. (1993). The theory of figural concepts. Educational Studies in Mathematics, 24(2), pp. 139-162.

Forman, E. (2014). Communities of Practice in Mathematics Education. En S. Lerman (Ed.), Encyclopedia of Mathematics Education (pp. 78-81). Springer.

Freudenthal, H. (1991). Revisiting Mathematics Education. Kluwer Academic Publishers. 
Gazzetta, M. (1989). A modelagem como estratégia de aprendizagem da Matemática em cursos de aperfeiçoamento de professores. Tesis de maestría. Universidade Estadual Paulista.

Gee, J. P. (2008). Learning and Games. En K. Salen (Ed.), The Ecology of Games. Connecting Youth, Games, and Learning (pp. 21-40). Cambridge: The MIT Pres.

Hanna, G. y De Villiers, M. (Eds.). (2012). Proof and Proving in Mathematics Education. Springer.

Kaiser, G (Ed.) (2017). Proceedings of the 13th International Congress on Mathematical Education. Springer.

Lakatos, I. (1978). Pruebas y refutaciones. La lógica del descubrimiento matemático. Alianza.

Larrosa, J. (2003). Entre las lenguas. Lenguaje y educación después de Babel. Laertes.

Ministerio de Educación Ciencia y Tecnología. (2011). NAP. Tercer ciclo. https://www.educ. ar/recursos/110570/nap-secundaria-matematica

Ministerio de Educación de la provincia de Santa Fe. (2014). Diseño Curricular. Educación Secundaria Orientada de la Provincia de Santa Fe.

Ouvrier-Buffet, C. (2015) A model of mathematicians' approach to the defining processes. K. Krainer y N. Vondrová. CERME 9 Proceedings of the Ninth Congress of the European Society for Research in Mathematics Education (pp. 2214-2220) Charles University in Prague.

Propuesta de estándares para la acreditación de las carreras de profesorado universitario en matemática. (2012). http://www.cin.edu.ar/comisiones/asuntos-academicos-material-en-tratamiento/subcomision-de-profesorados/

Ravn, O. y Skovsmose O. (2019). Connecting Humans to Equations. Springer.

Reid, D and Knipping, C. (2010). Proof in Mathematics Education. Research, Learning and Teaching. Sense Publishers.

Robutti, O., Cusi, A., Clark-Wilson, A., Jaworski, B., Chapman, O., Esteley, C., Goos, M., Isoda, M. y Joubert, M. (2016). ICME international survey on teachers working and learning through collaboration. ZDM The International Journal on Mathematics Education, 48(5), 651-690.

Sriraman. B. y Mousoulides, N. (2014). Quasi-empiricalReasoning (Lakatos). En S. Lerman (Ed.). Encyclopedia of Mathematics Education (pp. 511-513). Springer.

Stillman, G.S., Blum, W. y Kaiser, G. (Eds.). (2017). Mathematical Modelling and Applications. Crossing and Researching Boundaries in Mathematics Education. Springer.

Stylianides, A.J. y Harel, G. (Eds.). (2018). Advances in Mathematics Education Research on Proof and Proving. An International Perspective. Springer. 
María Florencia Cruz, Cristina Esteley y Sara Scaglia

Tall, D. (1989). Concept images, computers and curriculum change. For the Learning of Mathematics, 9(3), 37-42.

Zahar, J. (1989). Dicionário Básico de Filosofia. Rio de Janeiro.

MARÍA FLORENCIA CRUZ

Dirección: $\quad$ Urquiza 193310 D. Santa Fe (Santa Fe). CP: 3000. Argentina. 